\title{
Late rising right ventricular pacing lead threshold four years after implantation of a dual chamber pacemaker
}

\author{
H. Zaky ${ }^{*}$ H. El Zein, A. Al-Mulla
}

Dubai hospital, Dubai, UAE.

E-mail: *hzaky64@hotmail.com

Received 7 May 2011; revised 1 June 2011, accepted 13 June 2011.

\begin{abstract}
Late rising in pacing threshold is an uncommon complication of permanent pacing. Treatment with high dose systemic steroid could spare the patient another procedure. We report a case of late rising pacing threshold that responded to high dose systemic steroid which lowered the pacing threshold to one volt, then rose again to around 2 volts.
\end{abstract}

Keywords: Late Rising Pacing Threshold

\section{INTRODUCTION}

Late rising pacing threshold is an uncommon complication of pacemaker implantation. The use of systemic steroids to treat high pacing threshold has been suggested, and large doses of systemic steroids have been reported to result in decrement in pacing thresholds. However, when steroids are discontinued, the thresholds generally increase again $[1,2]$.

\section{HISTORY}

78 year old lady with a long-standing history of hypertension on regular treatment presented with a 20 day history of exertional dyspnoea. She had no history of chest pains, palpitations, dizziness or syncope. She had no orthopnoea or paroxysmal nocturnal dyspnoea. Clinical examination revealed: BP 188/57, pulse 35 beats per minute, normal audible heart sounds, no added heart sounds and no murmurs, clear chest, and bilateral carotid bruits. ECG: showed sinus rhythm with second degree heart block mobitz type 2 (2:1 block), Labs: normal cardiac markers, normal renal function with no electrolyte abnormalities. Echocardiography showed fair LV function (ejection fraction 50\%), LV diastolic dysfunction grade 1 , mild mitral regurgitation, mild aortic regurgitation, moderate tricuspid regurgitation, pulmonary artery pressure $25 \mathrm{mmhg}$, no pericardial effusion, no regional wall motion abnormalities. She underwent coro- nary \& carotid angiography which was normal. A dual chamber permanent pacemaker was inserted on 01 10-2005 with RV lead pacing threshold of $<0.5 \mathrm{~V}$ at the time of implant. Both leads were steroid eluting, active fixation, and continued to have regular follow up in the pacemaker clinic.

On 23-07-2009 she presented with dizziness and a heart rate between 35 - $40 \mathrm{bpm}$ with a wide complex escape rhythm and loss of pacemaker capture. The RV lead capture threshold was found to be high both in bipolar and unipolar configuration (3.75 V/0.4 MS) with acceptable sensitivity and impedance (sensed $\mathrm{R}$ wave $>$ $12.5 \mathrm{mv}$, and impedance $465 \Omega$ ). The battery was not depleted; there were no metabolic derangements, electrolyte disturbances or drug effects. A myocardial perfusion scan showed no evidence of ischemia or scar that might explain the rising pacing threshold. Chest $\mathrm{X}$ ray did not show any dislodgment. The possibilities were either microdislodgement or exit block; so we increased the output to maximum with good capture and we thought of implanting another RV lead. However, we tried systemic steroids first, and started high dose prednisolone $60 \mathrm{mg}$ od for 4 weeks at the end of which her RV threshold dropped to $2.2 \mathrm{~V} / 0.4 \mathrm{MS}$. We then started tapering of the prednisolone over another 4 weeks at the end of which her RV threshold dropped to $1 \mathrm{~V} / 0.4 \mathrm{MS}$. On follow up (1-11-2009), the RV threshold was found to have increased to $2.2 \mathrm{~V} / 0.4 \mathrm{MS}$, but had stabilized, when she was last seen on 15-03-2011 her RV threshold was found to be $2 \mathrm{~V} / 0.4 \mathrm{MS}$.

\section{DISCUSSION}

In this case, our use of systemic steroids prevented any interventional implantation of a new right ventricular lead, and this was in line with what other authors have reported $[1,3,4]$. we gave steroids for 4 weeks and tapered over another 4 weeks in comparison to nagatomo et al. who gave steroids for 5 months with similar results, also Ferraro et al. used azathioprime to decrease the dose 


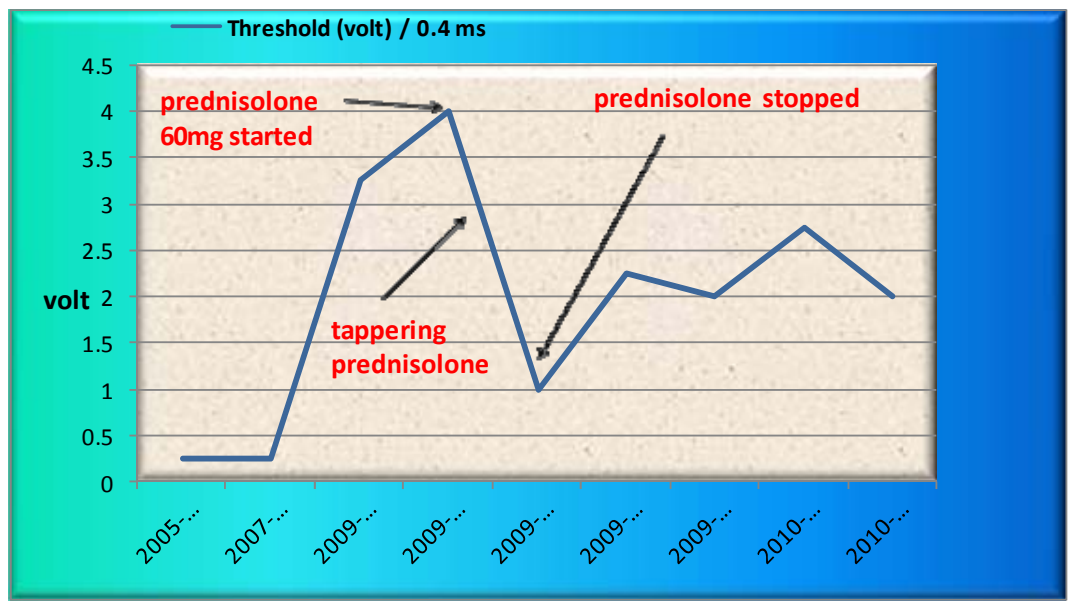

Figure 1. This graph shows the changes in right ventricular pacing threshold over time and decrease in threshold in response to steroids and the rise after discontinuation of the steroids.

DUBAI HOSPITAL

Name: MARYAM HASAN, ABOUL Date of exam.: 23/07/2009

Date of birth: 01/01/1932

Age: 77 Years 6 Months 22 Days

Examin. no.: 989/09
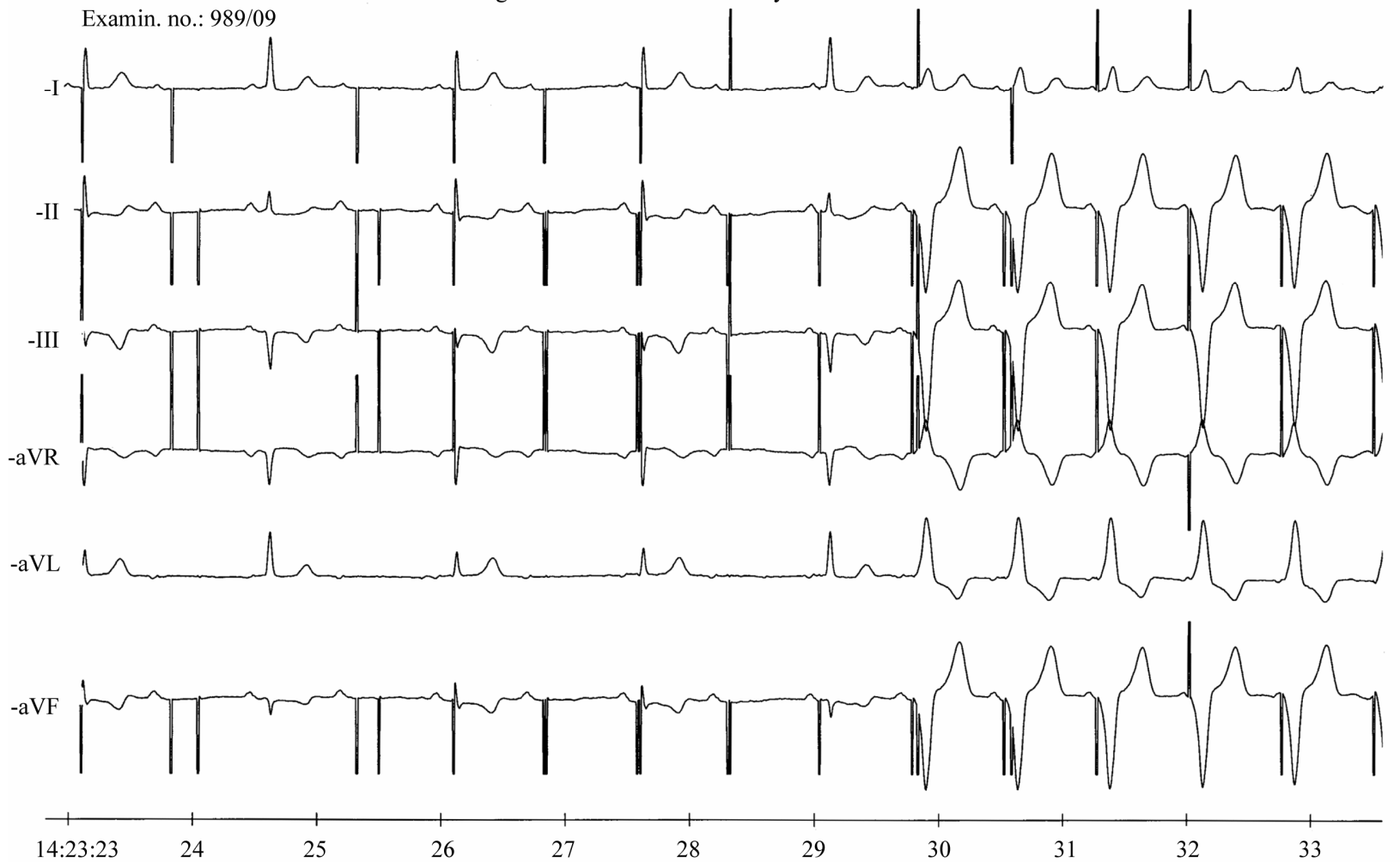

Figure 2. Showing intermittent capture of pacing before starting therapy.

of steroids to keep the pacing thresholds acceptable [5]. There are only few case reports and anecdotes of the use of steroids in late rising pacemaker lead thresholds. Despite the lack of randomized clinical trials to sup- port this pharmacological intervention, the use of systemic steroids was associated with the prevention of an intervention and decreased her threshold to $1 \mathrm{~V} / 0.4$ MS which rose to two volts after withdrawal of the 
steroids. This is consistent with other reports. [1-5].

\section{CONCLUSIONS}

In cases where pacemaker leads exhibit late rising thresholds, it is worth trying systemic steroids prior to subjecting patients to further intervention.

\section{REFERENCES}

[1] Beanland, D.S., Akyureckli, Y., Keon, W.J. (1979) Prednisolone in the management of exit block. Proceedings of the 6th World Symposium on Cardiac Pacing, Montreal, 2-5 October 1979, 18-3.

[2] Risby, O., Meibom, J., Nyboe, I., Schuller, H. (1981) The influence of prednisolone on pacemaker threshold (ab- stract). Pacing Clinical Electrophysiol, 4, A-68.

[3] Nagatomo, Y., Ogawa, T., et al. (1989) Pacing failure due to markedly increased stimulation threshold two years after implantation: Successful management with oral prednisolone: A case report. Pacing and Clinical Electrophysiology, 12, 1034-1037. doi:10.1111/j.1540-8159.1989.tb01922.x

[4] Preston, T.A., Judge, R.D., Lucchesi, B.R., et al. (1966) Myocardial threshold in patients in patients with artificial pacemakers. American Journal of Cardiology, 18, 83-89. doi:10.1016/0002-9149(66)90199-8

[5] Ferraro, A., Sabena Masi, A., Mazza, A. (2009) Increased stimulation threshold in a patient with autoimmune disease: Successful management with oral prednisolone and azathioprine. The European Journal of Pacing, 11, 527-529. 